\title{
Synthesis and Spectral Properties of Novel Thionaphtoquinone Dyes
}

\author{
Cigdem Sayil ${ }^{*}$ and Cemil Ibis \\ Istanbul University, Faculty of Engineering, Department of Chemistry, 34320 Avcllar-Istanbul, Turkey \\ *E-mail: sayil@istanbul.edu.tr \\ Received September 4, 2009, Accepted March 5, 2010
}

\begin{abstract}
2,3-Dichloro-1,4-naphtoquinone $\mathbf{1}$ compound was reacted with octadecanethiol $\mathbf{2}$ in two different mole ratio. Compound $\mathbf{3}$ was obtained from the reaction of $\mathbf{1}$ and $\mathbf{2}$ in 1:2 mole/mole ratio. Compounds $\mathbf{7}$ and $\mathbf{8}$ were obtained from reaction of $\mathbf{1}$ and $\mathbf{2}$ in 1:1 mole/mole ratio and known compound $\mathbf{9}$ was synthesized as by-product in this reaction. Novel compounds 5a-e were obtained from reaction of $\mathbf{1}$ and related thiols $4 \mathbf{a}-\mathbf{e}$. Known compounds $\mathbf{6 c}$ and $\mathbf{6 e}$ were synthesized as by-product in this reaction. The structures of the compounds were characterized by elemental analysis, UV-vis, FTIR, ${ }^{1} \mathrm{H}-\mathrm{NMR},{ }^{13} \mathrm{C}-\mathrm{NMR}$ and Mass spectroscopies.
\end{abstract}

Key Words: 1,4-Naphtoquinone, Disubstituethionaphtoquinone dyes, Michael reaction, Spectroscopic method

\section{Introduction}

Quinones, substituted quinones, bis-quinones were synthesized during the last century and their properties extensively studied. ${ }^{1-5}$ Their unique ability to attract electrons made them a desirable target in the fast growing fields of moleculer electronics and organic semiconductors. ${ }^{6}$ Moreover, different ainon radicals of more complex quinonic compounds exhibit intense absorbtion in the visible or near-infrared (NIR) region. Such a property is of importance in the fields of communication devices, ${ }^{7}$ optical storage ${ }^{8}$ and organic nonlinear optical (NLO) materials. ${ }^{9}$ Our recent work was on the synthesis of symmetrical and non-symmetrical thioquinonyl derivatives. ${ }^{4,5}$

This paper describes the synthesis of new members of a series of alkylmercapto-1,4-naphthoquinones by a MichaelType addition reaction and their spectral properties.

\section{Results and Discussion}

In this study we designed a synthesis of symmetrical and non-symmetrical naphtoquinonyl derivatives through which the quinone can be bridged via a thiolate moiety (Scheme 1).

Synthesis of naphtoquinonyl derivatives were carried out similarly to the method described. ${ }^{10}$ The ${ }^{1} \mathrm{H}-\mathrm{NMR}$ spectra $\left(\mathrm{CDCl}_{3}\right)$ of the symmetrical molecules $\mathbf{3}, \mathbf{6 c}$ and $\mathbf{6 e}$ exhibits doubledoublets of AA'BB' spin systems characteristic of two different para disubstituted aromatic compounds. The four naphthoquinone protons show chemical shifts at $\delta_{\mathrm{H}} 7.58-7.67(2 \mathrm{H})$ and $7.95-8.04(2 \mathrm{H})$ as double-doublets of AA'BB' system. The hydrogen atoms of the methylene group $\left(\mathrm{S}-\mathrm{CH}_{2}\right)$ adjacent to the sulfur atom were shifted to a lower field and display as triplet at $\delta_{\mathrm{H}} 3.17-3.28$. In the ${ }^{13} \mathrm{C}$-NMR APT spectra $\left(\mathrm{CDCl}_{3}\right)$ of the symmetrical molecules $\mathbf{3}, \mathbf{6 c}$ and $\mathbf{6 e}$, only one $\mathrm{C}$ signal of two $\mathrm{C}=\mathrm{O}$ groups appeared at $\delta_{\mathrm{c}} 178-179$ and only two $\mathrm{C}$ signals of four quaternary naphthoquinone carbons resonated at $\delta_{\mathrm{c}} 146-$ 148 and 132.3-133.2 and two $C$ signals of four vinylic naphthoquinone carbons appeared at $\delta_{\mathrm{c}} 132.3$ and 125 . Compound $\mathbf{6 c}$ and 6 e were known compounds earlier but their spectroscopic properties weren't investigated in detail.
The ${ }^{1} \mathrm{H}-\mathrm{NMR}$ spectra $\left(\mathrm{CDCl}_{3}\right)$ of the nonsymmetrical molecules 5a-f, 7, 8 and $\mathbf{9}$ similar shifts and splitting patterns at aromatic region. The four naphthoquinone protons show chemical shifts at $\delta_{\mathrm{H}} 7.64-7.75(2 \mathrm{H})$ and $8.04-8.14(2 \mathrm{H})$ as double-doublets of AA'BB' system. The hydrogen atoms of the methylene group $\left(\mathrm{S}-\mathrm{CH}_{2}\right)$ adjacent to the sulfur atom were shifted to a lower field and display as triplet at $\delta_{\mathrm{H}} 3.17-3.28$. In addition, In the ${ }^{1} \mathrm{H}-$ NMR spectra of $\mathbf{7}$ and $\mathbf{9}$, the hydrogen atoms of the methylene group $\left(\mathrm{O}-\mathrm{CH}_{2}\right)$ adjacent to the oxygen atom were shifted a quartet at $\delta_{\mathrm{H}} 4.46$ and 4.63 . In the ${ }^{13} \mathrm{C}-\mathrm{NMR}$ APT spectra $\left(\mathrm{CDCl}_{3}\right)$ of the nonsymmetrical molecules 5a-f, 7, 8 and $\mathbf{9}$ not similar to symmetric molecules. The nonsymmetrical molecules exhibit two $\mathrm{C}$ signal of two $\mathrm{C}=\mathrm{O}$ groups appeared at $\delta_{\mathrm{c}} 178.8-181.4$ and four $\mathrm{C}$ signals of four quaternary naphthoquinone carbons resonated at $\delta_{\mathrm{c}} 131-156$ and and four $\mathrm{C}$ signals of four vinylic
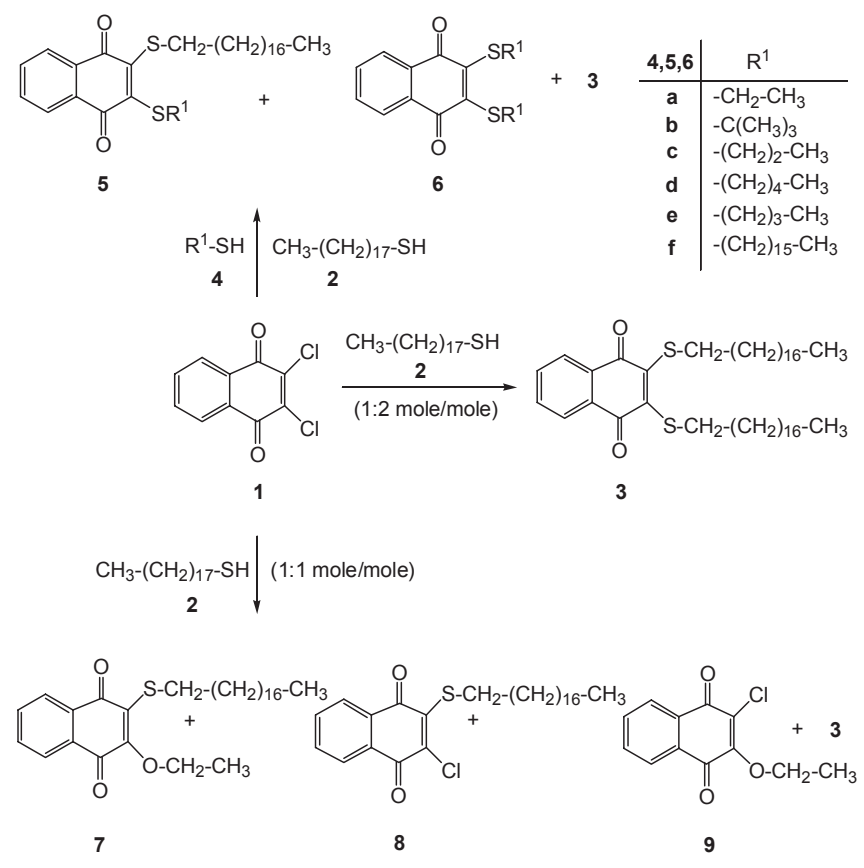

Scheme 1 
naphthoquinone carbons appeared at $\delta_{\mathrm{c}}$ 125-132. Compound 9 was known compounds earlier but their spectroscopic properties weren't investigated in detail.

In this study, we investegated solvent effect of synthesized dyes on the theirs absorption spectra by use of solvatochromism method. The absorption spectra of compounds $\mathbf{3}, \mathbf{5}, \mathbf{6 a - e}$ and $\mathbf{7 b}$ were measured in the nonpolar solvent 1,4-dioxane and in the polar solvents $\mathrm{CHCl}_{3}$ and $\mathrm{CH}_{2} \mathrm{Cl}_{2}$. The molar absorption coefficients were determined in the solvents and the data are presented in Table 1. As seen that, in the absorption spectra of the compound 5, 7, 8 and 9 were observed considerable differences depending on the substituent in the naphtoquinone fragment. Compounds 5 and 7 having electron-donor groups (RS and EtO) were absorbed at the longest wavelength, $\lambda_{\max }=473$ and $461 \mathrm{~nm}$ in the $\mathrm{CHCl}_{3}$. Compounds 8 and 9 having electronacceptor substituent $(\mathrm{Cl})$ were absorbed at the shortest wavelength $\lambda_{\max }=440$ and $335 \mathrm{~nm}$. in the $\mathrm{CH}_{2} \mathrm{Cl}_{2}$ (Fig. 1). ${ }^{11}$ The synthesized dyes $(\mathbf{3}, \mathbf{5 a}, \mathbf{b}-\mathbf{f}, \mathbf{6 e}, \mathbf{8})$ show the characteristic long wavelength thio-substituted quinonoid $\pi-\pi^{*}$ broad transition at $432 \sim 471 \mathrm{~nm}$.

\section{Experimental}

Melting points were measured on a Büchi B-540 melting point apparatus and are uncorrected. Elemental analyses were

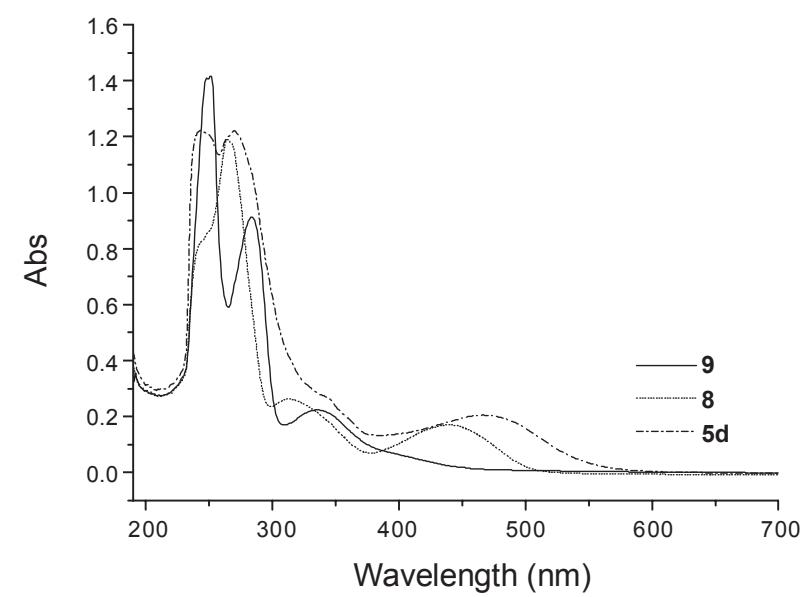

Figure 1. The absorbtion spectra of $\mathbf{5 d}, \mathbf{8}$ and $\mathbf{9}$ in $\mathrm{CH}_{2} \mathrm{Cl}_{2}$ solution. performed with a Carlo Erba 1106 Elemental analyzer. Infrared (IR) spectra were recorded in $\mathrm{KBr}$ pellets in Nujol mulls on a Shimadzu FTIR-8101 spectrometry. NMR spectra were record-

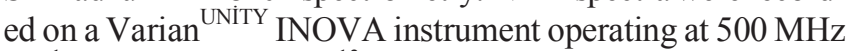
for ${ }^{1} \mathrm{H}$ and $125 \mathrm{MHz}$ for ${ }^{13} \mathrm{C}$. Mass spectra were obtained on a Thermo Finnigan LCQ Advantage MAX LC/MS spectrometer according to ESI probe. UV spectra in chloroform, dichloromethane and 1,4-dioxan solution were recorded on UV/vis Spectrometer TU-1901.

All chemicals and solvents were obtained commercially and used without purification. Products were isolated by column chromatography on $\mathrm{SiO}_{2}$ (Fluka Kieselgel 60, particle size 63 $200 \mu \mathrm{m})$. TLC plates: silica 60F 254 (Merck, Darmstadt), detection with ultraviolet light (254 $\mathrm{nm})$.

\section{Preparations.}

General procedure for preparation of thiosubstituted naphthoquinones: ${ }^{10}$ Sodium carbonate was dissolved in ethanol and equimolar amounts of 2,3-dichloro-1,4-naphtoquinone (1) and thiols were added slowly. Without heating, the mixture was stirred for $24 \mathrm{hr}$. The colour of the solution quickly changed and the extent of the reaction was monitored by TLC. Chloroform $(30 \mathrm{~mL})$ was added to the reaction mixture. The organic layer was separated and washed with water $(4 \times 30 \mathrm{~mL})$, and dried with $\mathrm{Na}_{2} \mathrm{SO}_{4}$. The mixture was concentrated in vacuo, and the residue was extracted with dichloromethane using a Soxhlet extractor. After the solvent was evaporated the residue was purified by column chromatography on silica gel.

Synthesis.

Synthesis of 2,3-bis(octaadecylthio)-1,4-naphthoquinone (3): Compound 3 was synthesized from reaction of 1 (1 g, 4.4 mmol) with octadecanethiol $2(2.72 \mathrm{~g}, 8.8 \mathrm{mmol})$ in $1: 2(\mathrm{~m} / \mathrm{m})$ ratio according to this general procedure.

The yield was 2.9 g. (93.0\%), pink coloured solid, mp $84 \sim$ $86{ }^{\circ} \mathrm{C}\left(\right.$ from $\left.\mathrm{CCl}_{4}\right) . R_{f}\left(\mathrm{CCl}_{4}\right)=0.36$. Found (\%): C, 74.92; $\mathrm{H}$, 10.67; S, 8.45. $\mathrm{C}_{46} \mathrm{H}_{78} \mathrm{O}_{2} \mathrm{~S}_{2}$. Calculated (\%): C, 75.97; H, 10.81; $\mathrm{S} 8.81 . \mathrm{UV} / \mathrm{vis}\left(\mathrm{CHCl}_{3}\right), \lambda_{\max } / \mathrm{nm}\left(\lg \varepsilon_{\max }\right): 468(3.43), 274$ (4.27), 241 (4.34). IR (KBr): v/ $\mathrm{cm}^{-1}: 2850,2919(\mathrm{C}-\mathrm{H}) ; 1656(\mathrm{C}=\mathrm{O})$; $1590(\mathrm{C}=\mathrm{C}) .{ }^{1} \mathrm{HNMR}\left(\mathrm{CDCl}_{3}\right), \delta 0.88\left(\mathrm{t}, 6 \mathrm{H}, J=7.08 \mathrm{~Hz} ., \mathrm{CH}_{3}\right)$; 1.20-1.66 (m, 64H, SCH$\left.{ }_{2} \mathrm{CH}_{2}\right) ; 3.26$ (t, 4H, $J=7.56 \mathrm{~Hz}$., $\mathrm{SCH}_{2}$ ); 7.67 (AA'BB' system, dd, 2H, $J=8.79$ Hz., $\mathrm{CH}_{\text {napht }}$ ); 8.04 (A A'BB' system, dd, $\left.2 \mathrm{H}, J=9.27 \mathrm{~Hz} ., \mathrm{CH}_{\text {napht }}\right) .{ }^{13} \mathrm{C} \mathrm{NMR}\left(\mathrm{CDCl}_{3}\right)$, $\delta 13.0\left(-\mathrm{CH}_{3}\right) ; 21.60,27.50,27.70,28.10,28.20,28.30,28.50$,

Table 1. UV-visible absorption data for compounds 3, 5a,b-f, 6e, 7, 8 and 9

\begin{tabular}{|c|c|c|c|}
\hline & \multicolumn{3}{|c|}{$\lambda_{\max }$ in $\mathrm{nm}(\log \varepsilon)$} \\
\hline & 1,4-Dioxane & $\mathrm{CHCl}_{3}$ & $\mathrm{CH}_{2} \mathrm{Cl}_{2}$ \\
\hline 3 & $461(3.38) 274(4.14) 243(4.09)$ & $468(3.43) 274(4.27) 241(4.34)$ & $464(3.75) 274(4.47) 240(4.46)$ \\
\hline 5a & $467(3.76) 282(4.44) 243(4.40)$ & $471(3.52) 274(4.26) 241(4.31)$ & $467(3.74) 278(4.42) 242(4.42)$ \\
\hline $5 c$ & $466(4.14) 281(4.80) 245(4.77)$ & $469(3.51) 273(4.28) 241(4.32)$ & $464(3.76) 270(4.34) 245(4.37)$ \\
\hline 5d & $465(3.74) 272(4.51) 243(4.48)$ & $473(3.52) 274(4.29) 241(4.34)$ & $467(3.70) 270(4.47) 244(4.47)$ \\
\hline $5 e$ & $467(3.65) 280(4.35) 243(4.32)$ & $470(3.56) 274(4.29) 241(4.32)$ & $467(3.59) 270(4.28) 245(4.30)$ \\
\hline $5 f$ & $467(3.62) 273(4.37) 242(4.33)$ & $471(3.53) 273(4.29) 241(4.31)$ & $467(3.55) 270(4.22) 248(4.23)$ \\
\hline $6 e$ & $464(3.74) 279(4.44) 243(4.40)$ & $469(3.37) 274(4.35) 242(4.38)$ & $467(3.60) 278(4.28) 244(4.26)$ \\
\hline 7 & $458(3.52) 276(4.33) 243(4.323$ & $464(3.47) 275(4.37) 241(4.37)$ & $460(3.86) 276(4.63) 246(4.55)$ \\
\hline 8 & $432(3.71) 265(4.59) 192(4.02)$ & $433(3.18) 260(4.46) 237(3.80)$ & $440(3.87) 313(4.06) 265(4.71)$ \\
\hline 9 & $281(4.27) 251(4.47)$ & & $335(3.85) 284(4.46) 252(4.65)$ \\
\hline
\end{tabular}


28.60, 28.65, 28.67, 28.69, 29.46, 30.92, 33.98, $38.30\left(-\mathrm{CH}_{2-}\right)$; 132.0, 125.0 ( $\left.\mathrm{CH}_{\text {napht. }}\right)$; 146.0, 132.3 ( $\left.\mathrm{C}_{\text {napht. }}\right) ; 178.0(\boldsymbol{C}=\mathrm{O})$. Found: $m / z 727.5\left[\mathrm{M}^{+}\right] . \mathrm{C}_{46} \mathrm{H}_{78} \mathrm{O}_{2} \mathrm{~S}_{2}$. Calculate: $\mathrm{M}=727.2$.

Synthesis of 2-(octadecylthio)-3-(ethylthio)-1,4-naphthoquinone (5a): Compound 5a was synthesized from reaction of $1(1 \mathrm{~g}, 4.4 \mathrm{mmol})$ with octadecanethiol $2(1.26 \mathrm{~g}, 4.4 \mathrm{mmol})$ and ethanethiol $4 \mathbf{a}(0.27 \mathrm{~g}, 4.4 \mathrm{mmol})$ according to this general procedure. In this reaction compound $\mathbf{3}$ was obtained as by-product.

The yield was 1.0 g. (45.0\%), deep bordeaux coloured solid, mp $83 \sim 84{ }^{\circ} \mathrm{C}$ (from $\left.\mathrm{CCl}_{4}\right) . R_{f}\left(\mathrm{CCl}_{4}\right)=0.31$. Found (\%): C, 71.79; H, 8.78; S, 12.83. $\mathrm{C}_{30} \mathrm{H}_{46} \mathrm{O}_{2} \mathrm{~S}_{2}$. Calculated (\%): C, 71.66; $\mathrm{H}, 9.22 ; \mathrm{S} 12.75$. UV/vis $\left(\mathrm{CHCl}_{3}\right), \lambda_{\max } / \mathrm{nm}\left(\lg \varepsilon_{\max }\right): 471$ (3.52), 274 (4.26), 241 (4.31). IR (KBr): v/ $\mathrm{cm}^{-1}: 2850,2917,2950$ $(\mathrm{C}-\mathrm{H}) ; 1653(\mathrm{C}=\mathrm{O}) ; 1589(\mathrm{C}=\mathrm{C}) .{ }^{1} \mathrm{H}$ NMR $\left(\mathrm{CDCl}_{3}\right), \delta 0.89(\mathrm{t}$, $6 \mathrm{H}, J=6.84 \mathrm{~Hz}$., $\left.\mathrm{CH}_{3}\right) ; 1.24-1.66\left(\mathrm{~m}, 32 \mathrm{H}, \mathrm{SCH}_{2} \mathrm{CH}_{2}\right) ; 3.30$ (t, 4H, $J=7.56$ Hz., SCH ) $^{2} 7.68$ (AA'BB' system, dd, 2H, $J=$ $8.78 \mathrm{~Hz} ., \mathrm{CH}_{\text {napht }}$ ); 8.04 (AA'BB' system, dd, $2 \mathrm{H}, J=9.27 \mathrm{~Hz}$., $\left.\mathrm{CH}_{\text {napht }}\right) .{ }^{13} \overline{\mathrm{CNMR}}\left(\mathrm{CDCl}_{3}\right), \delta 13.0,15.26\left(-\mathrm{CH}_{3}\right) ; 22.86,24.08$, 28.91, 29.32, 29.54, 29.67, 29.76, 29.83, 29.85, 29.89, 30.67, $32.12,35.20,37.11\left(-\mathrm{CH}_{2}-\right) ; 127.0,127.02,133.58\left(\mathrm{CH}_{\text {napht. }}\right)$; 133.30, 133.51, 147.93, 148.24 ( $\left.\mathrm{C}_{\text {napht. }}\right) ; 179.14,179.17(\boldsymbol{C}=\mathrm{O})$. Found: $m / z 503.24\left[\mathrm{M}^{+}\right] . \mathrm{C}_{20} \mathrm{H}_{46} \mathrm{O}_{2} \mathrm{~S}_{2}$. Calculated: $\mathrm{M}=502.82$.

Synthesis of 2-(octadecylthio)-3-(t-butylthio)-1,4-naphthoquinone (5b): Compound $\mathbf{5 b}$ was synthesized from reaction of 1 (1 g, $4.4 \mathrm{mmol}$ ) with octadecanethiol 2 (1.26 g, $4.4 \mathrm{mmol})$ and $t$-butanethiol $4 \mathbf{b}(0.4 \mathrm{~g}, 4.4 \mathrm{mmol})$ according to this general procedure. In this reaction compound $\mathbf{3}$ was obtained as byproduct.

The yield was 1.2 g. (51.2\%), red coloured solid, mp $58 \sim$ $59{ }^{\circ} \mathrm{C}\left(\right.$ from $\left.\mathrm{CCl}_{4}\right) . R_{f}\left(\mathrm{CCl}_{4}\right)=0.31$. Found (\%): C, 72.02; H, 9.49. $\mathrm{C}_{32} \mathrm{H}_{50} \mathrm{O}_{2} \mathrm{~S}_{2}$. Calculated (\%): C, 72.40; H, 9.49. IR (KBr): v/cm ${ }^{-1}: 2848,2922,2954(\mathrm{C}-\mathrm{H}) ; 1666(\mathrm{C}=\mathrm{O}) ; 1591(\mathrm{C}=\mathrm{C}) .{ }^{1} \mathrm{H}$ $\operatorname{NMR}\left(\mathrm{CDCl}_{3}\right), \delta 0.88\left(\mathrm{t}, 12 \mathrm{H}, J=6.83 \mathrm{~Hz} ., \mathrm{CH}_{3}\right) ; 1.21-1.66$ $\left(\mathrm{m}, 32 \mathrm{H}, \mathrm{SCH}_{2} \mathrm{CH}_{2}\right) ; 3.30$ (t, $2 \mathrm{H}, J=7.56 \mathrm{~Hz}$., $\left.\mathrm{SCH}_{2}\right) ; 7.67-7.73$ (m, 2H, $\left.\mathrm{CH}_{\text {napht }}\right)$; 8.04-8.11 (m, 2H, $\left.\mathrm{CH}_{\text {napht }}\right) .{ }^{13} \mathrm{CNMR}\left(\mathrm{CDCl}_{3}\right)$, $\delta 14.29,1 \overline{6} .11,22.87,32.13\left(-\mathrm{CH}_{3}\right) ; \overline{28} .93,28.99,29.02,29.53$, 29.55, 29.67, 29.90, 30.46, 30.76, 31.65, 32.63, 33.33, 35.36, 52.69 (-CH $\left.2^{-}\right)$; 70.1 (-S-C-); 133.58, 133.69, 133.74, 134.0 $\left(\underline{\mathrm{CH}}_{\text {napht. }}\right) ; 132.82,133.33,141.08,161.78$ ( C $\left._{\text {napht. }}\right) ; 179.11$, $181.14(\boldsymbol{C}=\mathrm{O})$. Found: $m / z 530.95\left[\mathrm{M}^{+}\right] . \mathrm{C}_{32} \mathrm{H}_{50} \mathrm{O}_{2} \mathrm{~S}_{2}$. Calculated : $\mathrm{M}=530.88$.

Synthesis of 2-(octadecylthio)-3-(propylthio)-1,4-naphthoquinone (5c) and 2,3-bis (propylthio)-1,4-naphthoquinone (6c): Compound $\mathbf{5 c}$ and $\mathbf{6 c}$ were synthesized from reaction of $\mathbf{1}(1 \mathrm{~g}$, $4.4 \mathrm{mmol}$ ) with octadecanethiol 2 (1.26 g, $4.4 \mathrm{mmol})$ and propanethiol $4 \mathbf{c}(0.33 \mathrm{~g}, 4.4 \mathrm{mmol})$ according to this general procedure. In this reaction compound $\mathbf{3}$ was obtained as by-product.

The yield was $0.5 \mathrm{~g}$. (21.4\%), pink coloured solid, mp $71 \sim$ $72{ }^{\circ} \mathrm{C}$ (from $\left.\mathrm{CCl}_{4}\right) . R_{f}\left(\mathrm{CCl}_{4}\right)=0.50$. Found $(\%): \mathrm{C}, 71.17 ; \mathrm{H}$, 9.50. $\mathrm{C}_{31} \mathrm{H}_{48} \mathrm{O}_{2} \mathrm{~S}_{2}$. Calculated (\%): C, 72.04; H, 9.36. UV/vis $\left(\mathrm{CHCl}_{3}\right), \lambda_{\max } / \mathrm{nm}\left(\lg \varepsilon_{\max }\right): 469$ (3.51), 273 (4.28), 241 (4.32). IR (KBr): v/cm $\mathrm{cm}^{-1}$ : 2850, 2917, $2950(\mathrm{C}-\mathrm{H}) ; 1655(\mathrm{C}=\mathrm{O}) ; 1589$ $(\mathrm{C}=\mathrm{C}) .{ }^{1} \mathrm{HNMR}\left(\mathrm{CDCl}_{3}\right), \delta 0.86\left(\mathrm{t}, 6 \mathrm{H}, J=6.84 \mathrm{~Hz} ., \mathrm{CH}_{3}\right) ; 1.21$ $1.65\left(\mathrm{~m}, 34 \mathrm{H}, \mathrm{SCH}_{2} \mathrm{CH}_{2}\right) ; 3.27$ (t, 4H, $J=6.84 \mathrm{~Hz}$., $\left.\mathrm{SCH}_{2}\right) ; 7.66$ (AA'BB' system, dd, 2H, $J=9.28$ Hz., $\mathrm{CH}_{\text {napht }}$ ); 8.04 (AA'BB' system, dd, $\left.2 \mathrm{H}, J=8.78 \mathrm{~Hz} ., \mathrm{CH}_{\text {napht }}\right) .{ }^{\overline{13}} \mathrm{C} \mathrm{NMR}\left(\mathrm{CDCl}_{3}\right), \delta$ 13.53, $14.26\left(-\mathrm{CH}_{3}\right) ; 22.87,24.08,28.91,29.54,29.67,29.76$, 29.83, 29.85, 29.89, 30.67, 32.12, 35.20, $37.11\left(-\mathrm{CH}_{2}-\right)$; 127.0,
127.15, $133.45\left(\mathrm{CH}_{\text {napht. }}\right)$; 133.30, 133.51, 147.91, 148.24 ( $\left.\mathrm{C}_{\text {napht. }}\right)$; 179.15, $179.17(\boldsymbol{C}=\mathrm{O})$. Found: $m / z 517.22\left[\mathrm{M}^{+}\right] . \mathrm{C}_{31} \mathrm{H}_{48} \mathrm{O}_{2} \mathrm{~S}_{2}$. Calculate: $\mathrm{M}=516.85$.

The yield was 0.4 g. (27.5\%), red coloured solid, mp 54 $55^{\circ} \mathrm{C}$ (lit. $\left.{ }^{2} 56{ }^{\circ} \mathrm{C}\right) ; R_{f}\left(\mathrm{CCl}_{4}\right)=0.26$. Found $(\%): \mathrm{C}, 63.20 ; \mathrm{H}$, 6.54. $\mathrm{C}_{31} \mathrm{H}_{48} \mathrm{O}_{2} \mathrm{~S}_{2}$. Calculated (\%): C, 62.71; H, 5.92. IR (KBr): v/cm ${ }^{-1}: 2857,2927,2958(\mathrm{C}-\mathrm{H}) ; 1658(\mathrm{C}=\mathrm{O}) ; 1590(\mathrm{C}=\mathrm{C}) .{ }^{1} \mathrm{H}$ $\operatorname{NMR}\left(\mathrm{CDCl}_{3}\right), \delta$ 0.78-0.96 (t, $\left.6 \mathrm{H}, J=6.84 \mathrm{~Hz} ., \mathrm{CH}_{3}\right) ; 1.15-1.62$ (m, 44H, $\left.\mathrm{SCH}_{2} \mathrm{CH}_{2}\right) ; 3.17$ (t, 4H, J=7.32 Hz., $\left.\mathrm{SCH}_{2}\right) ; 7.59$ (AA'BB' system, dd, 2H, $J=8.78 \mathrm{~Hz} . \mathrm{CH}_{\text {napht }}$ ); 7.94 (AA'BB' system, dd, $\left.2 \mathrm{H}, J=9.27 \mathrm{~Hz} ., \mathrm{CH}_{\text {napht }}\right) .{ }^{13} \mathrm{C} \mathrm{NMR}\left(\mathrm{CDCl}_{3}\right), \delta 14.29$ $\left(-\mathrm{CH}_{3}\right) ; 33.30,37.10\left(-\mathrm{CH}_{2}-\right) ; \overline{12} 6.76,133.56\left(\mathrm{CH}_{\text {napht. }}\right) ; 133.25$, 148.05 ( $\left.\mathrm{C}_{\text {napht. }}\right) ; 179.13(\boldsymbol{C}=\mathrm{O})$. Found: $m / z 306.96\left[\mathrm{M}^{+}\right] . \mathrm{C}_{16}$ $\mathrm{H}_{18} \mathrm{O}_{2} \mathrm{~S}_{2}$. Calculate: $\mathrm{M}=306.45$.

Synthesis of 2-(octadecylthio)-3-(penthylthio)-1,4-naphthoquinone (5d): Compound $\mathbf{5 d}$ was synthesized from reaction of 1 (1 g, $4.4 \mathrm{mmol}$ ) with octadecanethiol 2 (1.26 g, $4.4 \mathrm{mmol})$ and pentanethiol $4 \mathbf{d}(0.46 \mathrm{~g}, 4.4 \mathrm{mmol})$ according to this general procedure. In this reaction compound $\mathbf{3}$ was obtained as byproduct.

The yield was $0.7 \mathrm{~g}$. (27.9\%), pink coloured solid, mp $65 \sim$ $66{ }^{\circ} \mathrm{C}\left(\right.$ from $\left.\mathrm{CCl}_{4}\right) \cdot R_{f}\left(\mathrm{CCl}_{4}\right)=0.64$. Found $(\%)$ : C, 72.81; $\mathrm{H}$, 9.04. $\mathrm{C}_{33} \mathrm{H}_{52} \mathrm{O}_{2} \mathrm{~S}_{2}$. Calculated (\%): C, 72.79; H, 8.17. UV/vis $\left(\mathrm{CHCl}_{3}\right), \lambda_{\max } / \mathrm{nm}\left(\lg \varepsilon_{\max }\right): 473$ (3.52), 274 (4.29), 241 (4.34). IR (KBr): v/cm ${ }^{-1}: 2850,2917,2950(\mathrm{C}-\mathrm{H}) ; 1657(\mathrm{C}=\mathrm{O}) ; 1592$ $(\mathrm{C}=\mathrm{C}) .{ }^{1} \mathrm{H}$ NMR $\left(\mathrm{CDCl}_{3}\right), \delta$ 0.86-0.90 (m, 6H, $\left.\mathrm{CH}_{3}\right) ; 1.24-1.67$ $\left(\mathrm{m}, 38 \mathrm{H}, \mathrm{SCH}_{2} \mathrm{CH}_{2}\right) ; 3.28$ (t, 4H, J=7.56 Hz., $\left.\mathrm{SCH}_{2}\right) ; 7.68$ (AA'BB' system, dd, 2H, $J=8.79 \mathrm{~Hz} ., \mathrm{CH}_{\text {napht }}$ ); 8.04 (AA'BB' system, dd, $\left.2 \mathrm{H}, J=9.27 \mathrm{~Hz} ., \mathrm{CH}_{\text {napht }}\right) .{ }^{\overline{13}} \mathrm{C} \mathrm{NMR}\left(\mathrm{CDCl}_{3}\right), \delta$ $14.07,14.26\left(-\mathrm{CH}_{3}\right) ; 22.40,22.87,29.67,29.76,29.83,29.84$, $29.85,29.86,29.89,30.35,30.68,31.10,32.12,35.16,35.20$ $\left(-\mathrm{CH}_{2}-\right) ; 127.01,133.53\left(\mathrm{CH}_{\text {napht. }}\right) ; 133.32,148.08,148.11$ ( C $\left._{\text {napht. }}\right) ; 179.21(\boldsymbol{C}=\mathrm{O})$. Found: $m / z 545.25\left[\mathrm{M}^{+}\right] . \mathrm{C}_{33} \mathrm{H}_{52} \mathrm{O}_{2} \mathrm{~S}_{2}$. Calculate: $\mathrm{M}=544.50$.

Synthesis of 2-(octadecylthio)-n-butylthio)-1,4-naphthoquinone (5e) and 2,3-bis (n-butylthio)-1,4-naphthoquinone (6e): Compound $5 \mathbf{e}$ and $\mathbf{6 e}$ were synthesized from reaction of $\mathbf{1}$ (1 g, $4.4 \mathrm{mmol}$ ) with octadecanethiol 2 (1.26 g, $4.4 \mathrm{mmol})$ and $n$-butanethiol $4 \mathrm{e}(0.40 \mathrm{~g}, 4.4 \mathrm{mmol})$ according to this general procedure. In this reaction compound $\mathbf{3}$ was obtained as byproduct.

The yield was 1.0 g. (43.0\%), pink coloured solid, mp $73 \sim$ $74{ }^{\circ} \mathrm{C}$ (from $\left.\mathrm{CCl}_{4}\right) \cdot R_{f}\left[\mathrm{CCl}_{4} / \mathrm{CH}_{2} \mathrm{Cl}_{2}(1: 1)\right]=0.73$. Found (\%): $\mathrm{C}$, 72.93; H, 9.73; S, 11.24. $\mathrm{C}_{32} \mathrm{H}_{50} \mathrm{O}_{2} \mathrm{~S}_{2}$. Calculated (\%): C, 72.40; H, 9.49; S, 12.07. UV/vis $\left(\mathrm{CHCl}_{3}\right), \lambda_{\max } / \mathrm{nm}\left(\lg \varepsilon_{\max }\right): 470$ (3.56), 274 (4.29), 241 (4.32). IR (KBr): v/cm ${ }^{-1}: 2850,2917,2950$ $(\mathrm{C}-\mathrm{H}) ; 1655(\mathrm{C}=\mathrm{O}) ; 1590(\mathrm{C}=\mathrm{C}) .{ }^{1} \mathrm{HNMR}\left(\mathrm{CDCl}_{3}\right), \delta$ 0.86-0.93 (m, 6H, $\left.\mathrm{CH}_{3}\right) ; 1.21-1.65$ (m, 36H, $\left.\mathrm{SCH}_{2} \mathrm{CH}_{2}\right) ; 3.27$ (m, 4H, $\mathrm{SCH}_{2}$ ); 7.68 (AA'BB' system, dd, $2 \mathrm{H}, J=9.27 \mathrm{~Hz}$., $\mathrm{CH}_{\text {napht }}$ ); 8.04 (AA'BB' system, dd, $\left.2 \mathrm{H}, J=9.28 \mathrm{~Hz} ., \mathrm{CH}_{\text {napht }}\right) .{ }^{13} \mathrm{C}$ NMR $\left(\mathrm{CDCl}_{3}\right), \delta$ 13.77, $14.24\left(-\mathrm{CH}_{3}\right) ; 22.07,22.86,28.92,29.32$, 29.53, 29.83, 29.67, 29.75, 29.84, 29.85, 29.88, 29.89, 30.68, $32.11,32.74,34.88,35.20\left(-\mathrm{CH}_{2}-\right) ; 127.0,133.43\left(\mathrm{CH}_{\text {napht. }}\right)$; 133.33, 133.52, 148.08, $148.14\left(\underline{C}_{\text {napht. }}\right) ; 179.20(\boldsymbol{C}=\mathrm{O})$. Found: $m / z 531.25\left[\mathrm{M}^{+}\right] . \mathrm{C}_{32} \mathrm{H}_{50} \mathrm{O}_{2} \mathrm{~S}_{2}$. Calculate: $\mathrm{M}=530.88$.

The yield was 0.3 g. (22.0\%), red coloured solid, mp $37{ }^{\circ} \mathrm{C}$ (lit. $\left.{ }^{2} 37{ }^{\circ} \mathrm{C}\right) ; R_{f}\left[\mathrm{CCl}_{4} / \mathrm{CH}_{2} \mathrm{CI}_{2}(1: 1)\right]=0.50$. Found (\%): $\mathrm{C}$, 65.46; H, 6.97. $\mathrm{C}_{18} \mathrm{H}_{22} \mathrm{O}_{2} \mathrm{~S}_{2}$. Calculated (\%): C, 64.63; H, 6.62. 
$\mathrm{UV} / \mathrm{vis}\left(\mathrm{CHCl}_{3}\right), \lambda_{\max } / \mathrm{nm}\left(\lg \varepsilon_{\max }\right): 469$ (3.37), 274 (4.35), 242 (4.38). IR (KBr): v/cm $\mathrm{cm}^{-1}$ : 2857, 2927, $2958(\mathrm{C}-\mathrm{H}) ; 1658(\mathrm{C}=\mathrm{O})$; $1589(\mathrm{C}=\mathrm{C}) .{ }^{1} \mathrm{H}$ NMR $\left(\mathrm{CDCl}_{3}\right), \delta$ 0.86-0.93 (m, 6H, $\left.\mathrm{CH}_{3}\right)$; $1.42-1.65\left(\mathrm{~m}, 8 \mathrm{H}, \mathrm{SCH}_{2} \mathrm{CH}_{2}\right) ; 3.28\left(\mathrm{t}, 4 \mathrm{H}, J=7.32 \mathrm{~Hz}, \mathrm{SCH}_{2}\right)$; 7.66 (AA'BB' system, dd, $2 \mathrm{H}, J=8.78 \mathrm{~Hz} ., \mathrm{CH}_{\text {napht }}$ ); 8.03 (AA'BB' system, dd, $\left.2 \mathrm{H}, J=9.27 \mathrm{~Hz} ., \mathrm{CH}_{\text {napht }}\right) .{ }^{13} \mathrm{C} \mathrm{NMR}$ $\left(\mathrm{CDCl}_{3}\right), \delta 13.80\left(-\mathrm{CH}_{3}\right) ; 33.30,34.86\left(-\mathrm{CH}_{2^{-}}\right) ; 126.74,133.53$ $\left(\mathrm{CH}_{\text {napht. }}\right) ; 133.25,148.02$ ( $\left.\mathrm{C}_{\text {napht. }}\right) ; 179.12(\boldsymbol{C}=\mathrm{O})$. Found: $\mathrm{m} / z$ $335.02\left[\mathrm{M}^{+}\right] . \mathrm{C}_{18} \mathrm{H}_{22} \mathrm{O}_{2} \mathrm{~S}_{2}$. Calculate: $\mathrm{M}=334.50$.

Synthesis of 2-(octadecylthio)-3-(hexadecylthio)-1,4-naphthoquinone (5f): Compound $\mathbf{5 f}$ was synthesized from reaction of 1 ( $1 \mathrm{~g}, 4.4 \mathrm{mmol})$ with octadecanethiol 2 (1.26 g, $4.4 \mathrm{mmol})$ and hexadecanethiol $\mathbf{4 f}(1.14 \mathrm{~g}, 4.4 \mathrm{mmol})$ according to this general procedure. In this reaction compound $\mathbf{3}$ was obtained as by-product.

The yield was 1.7 g. (56.5\%), orange coloured solid, mp 59 $60{ }^{\circ} \mathrm{C}$ (from $\left.\mathrm{CCl}_{4}\right) . R_{f}\left(\mathrm{CCl}_{4}\right)=0.36$. Found $(\%)$ : C, 75.62; $\mathrm{H}$, 10.33; S, 8.62. $\mathrm{C}_{44} \mathrm{H}_{74} \mathrm{O}_{2} \mathrm{~S}_{2}$. Calculated (\%): C, 75.58; H, 10.66; $\mathrm{S}, 9.17$. UV/vis $\left(\mathrm{CHCl}_{3}\right), \lambda_{\max } / \mathrm{nm}\left(\lg \varepsilon_{\max }\right): 471$ (3.53), 273 (4.29), 241 (4.31). IR (KBr): v/cm $\mathrm{cm}^{-1}: 2848,2915$ (C-H); 1666 $(\mathrm{C}=\mathrm{O}) ; 1589(\mathrm{C}=\mathrm{C}) .{ }^{1} \mathrm{H}$ NMR $\left(\mathrm{CDCl}_{3}\right), \delta 0.88(\mathrm{t}, 6 \mathrm{H}, J=6.84$ $\left.\mathrm{Hz}, \mathrm{CH}_{3}\right) ; 1.24-1.66\left(\mathrm{~m}, 60 \mathrm{H}, \mathrm{SCH}_{2} \mathrm{CH}_{2}\right) ; 3.26(\mathrm{t}, 4 \mathrm{H}, J=7.56$ Hz., $\mathrm{SCH}_{2}$ ); 7.67 (AA'BB' system, dd, $2 \mathrm{H}, J=8.79$ Hz., $\mathrm{CH}_{\text {napht }}$ ); 8.04 (AA'BB' system, dd, $2 \mathrm{H}, J=8.79 \mathrm{~Hz} ., \mathrm{CH}_{\text {napht }}$ ). ${ }^{13} \mathrm{CNMR}$ $\left(\mathrm{CDCl}_{3}\right), \delta 14.30\left(-\mathrm{CH}_{3}\right) ; 22.91,28.96,29.3 \overline{6}, 29.58,29.72$, 29.81, 29.88, 29.88, 29.89, 29.92, 30,70, 32.15, $35.21\left(-\mathrm{CH}_{2}^{-}\right)$; 127.0, $133.0\left(\mathrm{CH}_{\text {napht. }}\right)$; 133.50, 148.0 ( $\left.\mathrm{C}_{\text {napht. }}\right) ; 179.20(\boldsymbol{C}=\mathrm{O})$. Found: $m / z 699.42\left[\mathrm{M}^{+}\right] . \mathrm{C}_{44} \mathrm{H}_{74} \mathrm{O}_{2} \mathrm{~S}_{2}$. Calculate: $\mathrm{M}=699.21$.

Synthesis of 2-(octadecylthio)-3-(etoxy)-1,4-naphthoquinone (7), 2-(octadecylthio)-3-(choloro)-1,4-naphthoquinone (8) and 2-(choloro)-3-(etoxy)-1,4-naphthoquinone (9): Compound 7,8 and 9 were synthesized from reaction of 1 ( $1 \mathrm{~g}, 4.4$ mmol) with octadecanethiol $(1.26 \mathrm{~g}, 4.4 \mathrm{mmol})$ in $1: 1(\mathrm{~m} / \mathrm{m})$ ratio according to this general procedure. In this reaction compound 3 was obtained as by-product.

(7): The yield was 0.2 g. (7.2\%), red coloured solid, mp $61 \sim$ $62{ }^{\circ} \mathrm{C}\left(\right.$ from $\left.\mathrm{CCl}_{4}\right) . R_{f}\left(\mathrm{CCl}_{4}\right)=0.28$. Found $(\%): \mathrm{C}, 73.61 ; \mathrm{H}$, 9.56; S, 5.94. $\mathrm{C}_{30} \mathrm{H}_{46} \mathrm{O}_{3}$ S. Calculated (\%): C, 74.02; H, 9.50; $\mathrm{S}, 6.58 . \mathrm{UV} / \mathrm{vis}\left(\mathrm{CHCl}_{3}\right), \lambda_{\max } / \mathrm{nm}\left(\mathrm{lg} \varepsilon_{\max }\right): 464$ (3.47), 275 (4.37), 241 (4.37). IR (KBr): v/cm ${ }^{-1}: 2920,2952$ (C-H); 1661 $(\mathrm{C}=\mathrm{O}) ; 1590(\mathrm{C}=\mathrm{C}) .{ }^{1} \mathrm{H} \mathrm{NMR}\left(\mathrm{CDCl}_{3}\right), \delta 0.88(\mathrm{t}, 6 \mathrm{H}, J=7.08$ $\left.\mathrm{Hz}, \mathrm{CH}_{3}\right) ; 1.21-1.65\left(\mathrm{~m}, 32 \mathrm{H}, \mathrm{SCH}_{2} \mathrm{CH}_{2}\right) ; 3.17$ (t, 4H, $J=7.56$ $\mathrm{Hz}$., $\mathrm{SCH}_{2}$ ); 4.46 (q, 2H, J=7.32 Hz, $\mathrm{OCH}_{2}$ ); 7.67-8.05 (m, $\left.4 \mathrm{H}, \mathrm{CH}_{\text {napht }}\right) .{ }^{13} \mathrm{C} \mathrm{NMR}\left(\mathrm{CDCl}_{3}\right), \delta 13.09,14.89\left(-\mathrm{CH}_{3}\right) ; 27.72$, 28.44, 29.57, 28.63, 28.66, 28.68, 28.69, 29.21, 29.24, 29.33, $30.43,30.92,32.10\left(-\mathrm{CH}_{2}-\right) ; 68.88\left(\mathrm{OCH}_{2}-\right) ; 125.36,125.58$, 130.52, 132.52 ( $\left.\mathrm{CH}_{\text {napht. }}\right) ; 131.47,132.47,133.93,156.76$ $\left(\mathrm{C}_{\text {napht. }}\right) ; 177.79,181.7(\boldsymbol{C}=\mathrm{O})$. Found: $m / z 487.28\left[\mathrm{M}^{+}\right] . \mathrm{C}_{30}$
$\mathrm{H}_{46} \mathrm{O}_{3} \mathrm{~S}$. Calculate: $\mathrm{M}=486.76$.

(8): The yield was 0.9 g. (42.0\%), yellow coloured solid, mp $90 \sim 92{ }^{\circ} \mathrm{C}\left(\right.$ from $\left.\mathrm{CHCl}_{3}\right) . R_{f}\left(\mathrm{CHCl}_{3}\right)=0.77$. Found $(\%)$ : , 71.39; H, 8.03; S, 7.60. $\mathrm{C}_{28} \mathrm{H}_{41} \mathrm{O}_{2} \mathrm{SCl}$. Calculated (\%): C, 70.48; $\mathrm{H}, 8.66 ; \mathrm{S}, 6.72$. UV/vis $\left(\mathrm{CHCl}_{3}\right), \lambda_{\max } / \mathrm{nm}\left(\lg \varepsilon_{\max }\right): 433$ (3.18), 260 (4.46), 237 (3.80). IR (KBr): v/ $\mathrm{cm}^{-1}: 2849,2917,2953$ $(\mathrm{C}-\mathrm{H}) ; 1672(\mathrm{C}=\mathrm{O}) ; 1590(\mathrm{C}=\mathrm{C}) .{ }^{1} \mathrm{H} \mathrm{NMR}\left(\mathrm{CDCl}_{3}\right), \delta 0.89(\mathrm{t}$, $\left.3 \mathrm{H}, J=6.83 \mathrm{~Hz}, \mathrm{CH}_{3}\right) ; 1.25-1.69\left(\mathrm{~m}, 32 \mathrm{H}, \mathrm{SCH}_{2} \mathrm{CH}_{2}\right) ; 3.37(\mathrm{t}$, $4 \mathrm{H}, J=7.56 \mathrm{~Hz}$., $\mathrm{SCH}$ ); 7.70-7.75 (m, 2H, $\left.\mathrm{CH}_{\text {napht }}\right) ; 8.07-8.14$ $\left(\mathrm{m}, 2 \mathrm{H}, \mathrm{CH}_{\text {napht }}\right) .{ }^{13} \mathrm{C} \mathrm{NMR}\left(\mathrm{CDCl}_{3}\right), \delta 14.28\left(-\mathrm{CH}_{3}\right) ; 22.88$, $28.85,29.29,29.55,29.64,29.75,29.83,29.86,29.89,30,67$, $32.13,34.57\left(-\mathrm{CH}_{2}-\right) ; 127.40,127.49,133.93,134.26\left(\mathrm{CH}_{\text {napht. }}\right)$; 131.56, 131.90, 133.99, 149.67 ( $\left.\mathrm{C}_{\text {napht. }}\right) ; 175.25,180.10(\boldsymbol{C}=\mathrm{O})$. Found: $m / z 477.18\left[\mathrm{M}^{+}\right] . \mathrm{C}_{28} \mathrm{H}_{41} \mathrm{O}_{2} \mathrm{SCl}$. Calculate: $\mathrm{M}=477.15$.

(9): The yield was 0.2 g. (14.4\%), yellow coloured solid, mp $94 \sim 96{ }^{\circ} \mathrm{C}$ (lit. $\left.{ }^{1} 97 \sim 98{ }^{\circ} \mathrm{C}\right) . R_{f}\left(\mathrm{CCl}_{4}\right)=0.33$. Found $(\%)$ : $\mathrm{C}, 61.45 ; \mathrm{H}, 4.30 . \mathrm{C}_{12} \mathrm{H}_{9} \mathrm{O}_{3} \mathrm{Cl}$. Calculated (\%): C, 60.95; H, 3.83. UV/vis $\left(\mathrm{CHCl}_{3}\right), \lambda_{\max } / \mathrm{nm}\left(\lg \varepsilon_{\max }\right): 335$ (3.85), 284 (4.46), 252 (4.65). IR (KBr): v/cm $\mathrm{cm}^{-1}: 2937,2985,3086(\mathrm{C}-\mathrm{H}) ; 1677$ $(\mathrm{C}=\mathrm{O}) ; 1591(\mathrm{C}=\mathrm{C}) .{ }^{1} \mathrm{H}$ NMR $\left(\mathrm{CDCl}_{3}\right), \delta 1.47(\mathrm{t}, 6 \mathrm{H}, J=7.32$ $\left.\mathrm{Hz}, \mathrm{CH}_{3}\right) ; 4.63$ (q, 2H, $\left.J=7.32 \mathrm{~Hz}, \mathrm{OCH}_{2}\right) ; 7.70-8.14(\mathrm{~m}, 4 \mathrm{H}$, $\left.\mathrm{CH}_{\text {napht }}\right) .{ }^{13} \mathrm{C} \mathrm{NMR}\left(\mathrm{CDCl}_{3}\right), \delta 16.19\left(-\mathrm{CH}_{3}\right) ; 70.89\left(-\mathrm{O}-\mathrm{CH}_{2}-\right)$; 127.04, 127.13, 131.06, 131.37 ( $\left.\mathrm{CH}_{\text {napht. }}\right) ; 129.47,131.06,131.27$, 156.83 ( Cnapht. $_{\text {. }}$; 178.81, $179.75(\boldsymbol{C}=\mathrm{O})$. Found: $m / z 236.87\left[\mathrm{M}^{+}\right]$. $\mathrm{C}_{12} \mathrm{H}_{9} \mathrm{O}_{3} \mathrm{Cl}$. Calculate: $\mathrm{M}=236.65$.

Acknowledgments. This study was financially supported by the Research Fund of the University of Istanbul.

\section{References}

1. Fieser, L. F.; Brown, R. H. J. Am. Chem. Soc. 1949, 71, 3609.

2. Tjepkema, J. J. Recueil. 1952, 71, 853.

3. Porter, T. H.; Tsai, C. S.; Kishi, T.; Kishi, H.; Folkers, K. Acta Pharm. Suec. 1978, 15(2), 97.

4. Ibis, C.; Gunes, Z. O. Dyes and Pigments 2008, 77(1), 39.

5. Goksel, F. S.; Ibis, C.; Bayrak, N. A. Phosphorus, Sulfur and Silicon 2005, 180(8), 1961.

6. Bittner, S.; Temtsin, G.; Sason, Y. Synthesis 2000, 1084.

7. Koroteev, N. I.; Magnitskii, S. A.; Shubin, V. V.; Sokolyuk, N. T. Jpn. J. Appl. Phys. 1997, 36(1B), 424.

8. Kawai, S. H.; Gilat, S. L.; Ponsinet, R.; Lehn, J. M. Chem. Eur. J. 1995, 1, 285.

9. Marder, S. R., Interrante, L. V., Casper, L. A., Eds.; In Materials Chemistry, an Emerging Discipline; American Chemical Society: Washington, D. C. 1995; p 189.

10. Otsubo, T.; Nabuhara, Y.; Kanefuji, K.; Asu, Y.; Ogura, F. J. Phys. Org. Chem. 1988, 1, 275.

11. Bukhtoyarova, A. D.; Ektova, L. V.; Alekseev, S. N.; Beregovaya, I. V. Russ. J. Org. Chem. 2003, 39(9), 1309. 